\title{
Quarantine Decision due to Coronavirus Pandemic
}

\author{
Shahryar Sorooshian ${ }^{1 *}$ \\ ${ }^{1}$ University of Gothenburg, SWEDEN \\ ${ }^{\star}$ Corresponding Author: sorooshian@gmail.com \\ Citation: Sorooshian S. Quarantine Decision due to Coronavirus Pandemic. Electron J Gen Med. $2020 ; 17(4):$ em206. \\ https://doi.org/10.29333/ejgm/7862 \\ ARTICLE INFO \\ Received: 26 Mar. 2020 \\ Accepted: 28 Mar. 2020

\section{ABSTRACT} \\ These days the world is facing Coronavirus disease (COVID-19) pandemic. More or less, governments decide to \\ quarantine some of the cities. However, it would be a hard decision to decide which cities should be on the list of \\ quarantine due to the social, psychological and financial costs. This short letter is proposing a systematic \\ methodology to solve this decision making challenge in public health administration.
}

Keywords: Coronavirus, COVID-19, quarantine
These days the world is facing Coronavirus disease (COVID19) pandemic. More or less, governments decide to quarantine some of the cities. However, it would be a hard decision to decide which cities should be on the list of quarantine due to the social, psychological and financial costs. This short letter is proposing a systematic methodology to solve this decision making challenge in public health administration.

Decision trial and evaluation laboratory (DEMATEL) is one of the most used methodologies from operational research that could deal with direct and indirect interrelationships and cross infection among cities. Many articles attempt to teach DEMATEL methodology, such as Ali, et al. (1). DEMATEL can be applied to help decision-makers based on the cause and effect sources of COVID-19 infection.

Ali et al. (1) intensely explained six steps of DEMTEL for $n$ alternatives that are different cities for the quarantine decision. In step 1, the input data for the initial direct-relation matrix for DEMATEL, will be the qualitative approach to the primary statistics of the COVID-19 infected cases and their traveling information during the past 2 weeks (or maximum incubation period for COVID-19). Although the ranking scale for the initial direct-relation matrix is subject to the decision-makers' preference, scale of $0-3$, from $0=$ no influence to $3=$ strong direct influence, could be suggested to initiate the cross infection between cities. For example, if many COVID-19 cases of city $i$ have visited city $j$ during past 2 weeks, then the ranking scale in the cell $i, j$ of the initial direct-relation matrix will be 3 . Vice versa, if no COVID-19 cases of city $i$ have visited city $j$, then the ranking scale in the cell $i, j$ of the initial direct-relation matrix will be 0 .

Next, following the rest of DEMATEL steps and calculations, the impact relation map will be achieved in step 6. From the result, causes sources, or positive D-R from the calculations, are distributing the infection and top distributors (based on the highest value of $D-R$ ) should be quarantined.

\section{REFERENCES}

1. Ali SAM, Sorooshian S, Kie CJ. Modelling for causal interrelationships by DEMATEL. Contemporary Engineering Sciences 2016;9(9):403-412. https://doi.org/10.12988/ces. 2016.6214 